\title{
EL RIESGO BETA DE LOS FONDOS DE PENSIONES EN CHILE
}

\author{
Recibido: 15 de noviembre de 2013 • Aprobado: 15 de mayo de 2014 \\ Werner Kristjanpoller Rodríguez ${ }^{* *}$ \\ Manuel García Sobarzo ${ }^{* * *}$
}

\section{RESUMEN}

El presente artículo tiene por objetivo estimar el riesgo beta para los fondos de pensiones administrados por las administradoras de fondos de pensión en Chile para el período comprendido entre los años 2002 y 2012. Se analiza la caracterización, consistencia y estabilidad del riesgo beta de estos fondos, mediante las metodologías de métodos de los mínimos cuadrados, método Blume y método Vasicek. Se concluye que el índice beta es una buena medida para determinar qué tan riesgosa puede ser la inversión, lo que demuestra que los fondos de pensiones tienen un comportamiento más bien defensivo, dada la naturaleza del portafolio de inversión.

\section{PALABRAS CLAVE}

Fondos de pensión, beta, riesgo y retorno.

\section{CLASIFICACIÓN JEL}

G12, G23

\section{CONTENIDO}

1. Introducción; 2. Revisión de la literatura; 3. Administradoras de fondos de pensiones; 4. Metodología y datos; 5 . Análisis de resultados; 6. Conclusiones; Bibliografía; Anexos.

Artículo de investigación, realizado entre abril del 2012 hasta abril 2013 en el Departamen de Industrias de la Universidad Técnica Federico Santa María, Valparaíso, Chile.

** Ingeniero civil industrial, Universidad Técnica Federico Santa María, Valparaíso, Chile. Magíster en Gestión Empresarial, Universidad Técnica Federico Santa María, Santiago, Chile. Doctor en Ciencias Empresariales, Universidad Autónoma de Madrid, Madrid, España. Profesor jornada completa, Universidad Técnica Federico Santa María, Valparaíso, Chile. Dirección: Avenida España 1680, Valparaíso, Chile. Teléfono: +56 32 2654571. Correo Electrónico: werner.kristjanpoller@usm.cl.

*** Ingeniero civil electrónico, Universidad de la Frontera, Temuco, Chile. Magíster en Gestión Empresarial Universidad Técnica Federico Santa María, Santiago, Chile. Agente Antofagasta, Asociación Chilena de Seguridad, Antofagasta, Chile. Dirección: Avenida Grecia 840, Antofagasta, Chile. Teléfono: +56 55 2626218. Correo Electrónico: manuel.in@gmail.com. 


\section{BETA RISK EN THE CHILEAN PENSION FUNDS}

\section{ABSTRACT}

This article aims to estimate the beta risk of pension funds managed by pension fund managers in Chile for the 2002 - 2012 time period. The characterization, consistency and stability of the beta risk for these funds are analyzed with the help of the minimum squares, Blume and Vasicek methods. The results conclude that the beta index is a good measure to determine how risky can an investment be, which proves that pension funds tend to have a defensive behavior given the nature of the investment portfolios.

\section{KEY WORDS}

Pension funds, beta, risk and return

\section{JEL CLASSIFICATION}

G12, G23

\section{CONTENT}

1. Introduction; 2. Literature review; 3. Pension fund administrators; 4. Methodology and data; 5. Analysis of results, 6. Conclusions; Bibliography; Attachments.

\section{O RISCO BETA DOS FUNDOS DE PENSÕES NO CHILE}

\section{RESUMO}

O presente artigo tem por objetivo estimar o risco beta para os fundos de pensões administrados pelas administradoras de fundos de pensão no Chile para o período compreendido entre os anos 2002 e 2012. Analisa-se a caracterização, consistência e estabilidade do risco beta destes fundos, mediante as metodologias de métodos dos mínimos quadrados, método Blume e método Vasicek. Concluise que o índice beta é uma boa medida para determinar que tão perigosa pode ser o investimento, o que demonstra que os fundos de pensões têm um comportamento mais bem defensivo, dada a natureza do portfólio de investimento.

\section{PALAVRAS CHAVES}

Fundos de pensão, beta, risco e volta.

\section{CLASSIFICAÇÃO JEL}

G12, G23.

\section{CONTEÚDO}

1. Introdução; 2. Revisão da literatura; 3. Administradoras de fundos de pensões; 4. Metodologia e dados; 5 . Análise de resultados; 6. Conclusões; Bibliografia; Anexos. 


\section{INTRODUCCIÓN}

El riesgo y la rentabilidad son conceptos inherentes a cualquier inversión, incluso en aquellas denominadas de libre de riesgo. Al tomar decisiones de inversión siempre se ha de analizar estos dos factores fundamentales, que no son independientes entre sí, sino que debieran tener una relación positiva, o sea, a mayor riesgo la rentabilidad debiera ser mayor. El riesgo se puede descomponer en el riesgo sistemático o de mercado, y el riesgo no sistemático o específico. Para el caso de las carteras de inversiones, se debe estudiar el riesgo sistemático, ya que recoge la sensibilidad de la misma de la varianza de mercado.

Un caso particular y de especial importancia de aplicación de estos factores, tanto para inversores expertos como para los que no tienen conocimientos para invertir, son los fondos de pensión, los cuales no están exentos de riesgo y contienen el dinero asociado a la jubilación de los trabajadores. Es en este contexto que este estudio viene a aportar información sobre los fondos de pensiones del mercado chileno.

Los sistemas de fondos de pensiones operan en Chile desde al año 1980, con transacciones de más de ciento cincuenta mil millones de dólares, lo que equivale aproximadamente a un $60 \%$ del PIB nacional. Por ley todos los trabajadores chilenos están obligados a ahorrar para las pensiones de jubilación en un sistema privado de administradoras de fondos de pensión (AFP), quienes buscan, mediante portafolios de inversión, manejar e incrementar el patrimonio de los futuros pensionados.

Las AFP presentan desde el año 2002 diferentes portafolios de inversión caracterizados por el nivel de riesgo. Así se tienen los multifondos A, B, C, D y E, donde el primero es el que presenta una mayor exposición al riesgo, y último, la menor. Cabe mencionar que el fondo E no se considera en el presente estudio ya que el porcentaje de renta variable que puede poseer está entre el $0 \%$ y el $5 \%$, por lo que el riesgo de mercado es muy bajo.

Este trabajo busca determinar el riesgo sistemático de los diferentes fondos que manejan las AFP, a través de los coeficientes beta desde la creación de los multifondos y diferentes métodos de predicción de dicho parámetro. Parra ello se aplicarán tres metodologías: mínimos cuadrados ordinarios, método de Blume y método de Vasicek.

Un punto importante es observar cómo la turbulencia de los mercados durante la crisis financiera subprime y la posterior crisis asociada a países de la Comunidad Europea impactó en la administración de los fondos de pensiones. 
También, con los resultados obtenidos se podrá determinar la existencia de efecto rebaño o manada. Raddatz y Schmukler (2011) determinan que los fondos son administrados de manera similar entre las administradoras, dada la regulación existente para las AFP.

El artículo se compone de seis secciones. La primera sección es esta introducción. La segunda sección es la Revisión de Literatura donde se exponen diversos estudios realizados sobre el tema. Luego, se hace una reseña del Sistema de Administración de Fondos de Pensión de Chile. La sección Metodología explica la forma en la que se llevará a cabo el estudio. En la quinta sección, Análisis de Resultados, se muestran y estudian los resultados obtenidos de la aplicación de los modelos. Para finalizar, en la sección Conclusiones se muestran las principales evidencias, consecuencias y perspectivas que se pueden derivar de este estudio.

\section{REVISIÓN DE LA LITERATURA}

Markowitz (1952) establece que todo inversionista es adverso al riesgo, lo cual implica que para la toma de decisión realizará un análisis de retorno frente a riesgo; así se busca la alternativa de inversión que combine de mejor manera el mayor retorno con menor riesgo. Introduce el concepto de retorno y varianza, como medida de gestión del riesgo, lo cual aplica en un análisis de distribución normal.

Markowitz (1952) plantea que se pueden diversificar las inversiones, a través de una cartera de activos financieros de modo tal que el riesgo global sea el más bajo posible, lo que genera los fundamentos de los conceptos de teoría de portafolio y frontera eficiente.

El retorno esperado de un portafolio se define como la suma ponderada de los retornos esperados de cada instrumento según la proporción de inversión (1)

$$
E\left(R_{p}\right)=\sum_{i=1}^{n} x_{i} E\left(R_{i}\right)
$$

en donde $x_{i}$ es el porcentaje de participación del activo en el portafolio, y $\mathrm{E}\left(\mathrm{R}_{i}\right)$ el retorno esperado. Para el cálculo de la varianza del portafolio se requiere la varianza individual de cada instrumento y la correlación de las rentabilidades entre pares de ellos. Markowitz (1952) define la desviación estándar de los rendimientos de un portafolio como medida de riesgo (2).

$$
\sigma_{p}=\sqrt{\sum_{i=1}^{N} x_{i}^{2} \sigma_{i}^{2}+\sum_{i=1}^{N} \sum_{j=1}^{N} x_{i} x_{j} \sigma_{i j}}, i \neq j
$$


donde,

$\sigma_{\mathrm{p}}$ es la desviación estándar del portafolio,

$\sigma_{\mathrm{i}}$ es la desviación estándar del activo $i$ y $\sigma_{\mathrm{ij}}$ es la covarianza entre la rentabilidad del activo $i$ y del activo $j$.

De este modelo propuesto por Markowitz (1952), se generó una serie de investigaciones sobre la relación del riesgo y el retorno de un portafolio de inversiones, donde se deriva uno de los modelos más utilizados en los tiempos modernos, el modelo Capital Asset Pricing Model (CAPM) por Sharpe (1964), Litner (1965) y Mossin (1966). Este modelo estima la rentabilidad esperada de un activo riesgoso como una función del riesgo del activo, al cual denominó beta, que no es más que la pendiente de la regresión lineal entre la rentabilidad del activo frente a la rentabilidad de mercado. El beta entonces queda definido según 3 .

$$
\beta=\frac{\operatorname{Cov}(i, M)}{\operatorname{Var}(M)}
$$

donde $\operatorname{Cov}(\boldsymbol{i}, \boldsymbol{M})$ es la covarianza entre la rentabilidad del activo $i$ y el retorno del mercado, y $\operatorname{Var}(\boldsymbol{M})$ es la varianza de los retornos del mercado.

El riesgo siempre estará presente en cualquier tipo de inversión, incluso a través de la diversificación. Según la teoría de portafolio se puede minimizar, pero nunca eliminar. En general el riesgo de un activo se compone del riesgo diversificable o riesgo no sistemático, y del riesgo no diversificable o riesgo sistemático. El riesgo no sistemático está asociado a la variabilidad del activo en particular, el cual a través de la diversificación puede llevarse a una mínima expresión dentro del portafolio. Por otra parte, el riesgo sistemático es el riesgo de mercado, el cual no se puede eliminar, lo que lo convierte en el más importante, ya que el no sistemático se puede minimizar.

El modelo CAPM enuncia que la rentabilidad esperada para un activo será la tasa libre de riesgo, más una prima de riesgo (4). La prima de riesgo es beta veces la diferencia entre la esperanza de retorno del mercado y la tasa libre de riesgo. A la diferencia entre la esperanza de retorno del mercado y la tasa libre de riesgo se le conoce como prima de mercado.

donde

$$
E\left(R_{i}\right)=R_{f}+\beta_{i}\left(E\left(R_{M}\right)-R_{f}\right)
$$

$\mathrm{R}_{i}$ es el rendimiento esperado del activo $\mathrm{i}, \mathrm{R}_{f}$ es la tasa libre de riesgo, $E\left(\mathrm{R}_{\mathrm{M}}\right)$ es el rendimiento esperado del mercado, $\beta_{i}$ es el parámetro beta propio del activo i. 
Tanto el beta como el modelo CAPM tiene detractores, pero aún así son los más utilizados. Fernández (2005) demuestra que la predicción de rentabilidad con este modelo tiene un mayor valor predictivo en el mediano plazo. Por otro lado, Kothari y Shanken (1995) concluyen que beta es un indicador preponderante y y también que la variable tamaño es una variable significativa que mejora la valoración del rendimiento esperado, mientras que Fama y French (1992) habían demostrado que el tamaño y la relación precio-libro son importantes para la predicción de retornos.

Las investigaciones sobre la medición del riesgo beta son muy abundantes, dada la relevancia que tiene este indicador en el mundo de las inversiones. García y García (2009) analizan el riesgo beta de planes de pensión españoles para el período 1995-2003, a través de un estudio de la caracterización, estabilidad y métodos para predecir el beta. Concluyen que los betas son significativos y tienen un comportamiento de preferencia defensivo, dada la naturaleza del portafolio que conforman las inversiones de los planes de pensiones, pero también, depende del indicador de mercado que se utilice; las sensibilidades tienden a aumentar si se utiliza como proxy del mercado un índice que contenga renta fija, además de renta variable, sobre todo en los planes que tienen mayor cantidad de renta variable, y que la mejor forma para predecirlo es a través de mínimos cuadrados ordinarios.

Arrau y Chumacero (1998) analizan la relación entre el tamaño de los fondos de pensiones en Chile y el desempeño financiero, y concluyen que el poder predictivo de los betas deja de ser estadísticamente significativo cuando considera el tamaño, y es el tamaño en forma cuadrática el que explica las diferencias de rentabilidad.

Zurita y Jara (1999) comparan el desempeño de los fondos de pensiones según el desempeño ajustado por riesgo en el período enero de 1987 a septiembre de 1998 y concluyen que el Índice de Sharpe es el mejor ratio para medir el desempeño de los fondos y que los desempeños ajustados por riesgo entre períodos no superpuestos presentan correlaciones seriales positivas. Mittelstaedt y Olsen (2003) analizaron la administración de fondos de pensiones chilenos y concluyeron que los administradores no generan valor adicional.

Olivares (2005), analiza los fondos de pensión entre 1997 y 2001 y concluye que el índice benchmark explica el 99 \% de la variabilidad de los retornos de los fondos y que las regulaciones existentes para los administradores de los fondos de pensión los llevan a generar el efecto manada.

Opazo, Raddatz y Schmukler (2009) analizan la demanda por instrumentos de largo plazo en Chile, administrados por inversionistas institucionales chilenos, tales como fondos mutuos, fondos de pensiones y compañías de seguros, mediante una 
comparación con las estructuras de plazos con la de los fondos mutuos en bonos de Estados Unidos. Concluye que las instituciones administradoras de activos chilenas, entre ellas las AFP, mantienen un porcentaje alto en activos de corto plazo en comparación con los fondos mutuos americanos, lo cual podría tener una explicación en minimizar el riesgo inflacionario.

Santillán, López y Montenegro (2010) concluyen que el papel de las AFP en el mercado de capitales chileno ha sido sustantivo en la madurez y consolidación.

Raddatz y Schmukler (2011) analizan el detalle de las inversiones de las administradoras de fondos de pensión en Chile para el período julio 1996 a diciembre 2005 de manera mensual, y encuentran evidencia de un comportamiento de rebaño o manada.

\section{ADMINISTRADORAS DE FONDOS DE PENSIONES}

Las AFP son sociedades anónimas que tienen por objeto exclusivo la administración del dinero destinados por los trabajadores para las futuras pensiones.

En el año 1980, se publicó en Chile el Decreto de Ley 3500 que cambió el antiguo sistema de pensiones por un sistema de capitalización individual. Las AFP reciben de cada cotizante, en forma mensual y obligatoria por ley, el $10 \%$ del sueldo imponible del afiliado. Este dinero lo administran con el objetivo de obtener rentabilidades ajustadas al riesgo.

Los cotizantes pueden elegir colocar los ahorros que tienen para la jubilación en cinco multifondos, los que se rigen según restricciones de inversiones en renta fija y renta variable (tabla 1). Se definen los fondos A, B, C, D y E, donde el fondo A es el más riesgoso y el fondo E el de menor riesgo.

Tabla 1. Límites vigentes de inversión en renta variable de los multifondos chilenos.

\begin{tabular}{|l|c|c|}
\cline { 2 - 3 } \multicolumn{1}{c|}{} & Limite máximo & Límite mínimo \\
\hline Fondo A & $80 \%$ & $40 \%$ \\
\hline Fondo B & $60 \%$ & $25 \%$ \\
\hline Fondo C & $40 \%$ & $15 \%$ \\
\hline Fondo D & $20 \%$ & $5 \%$ \\
\hline Fondo E & $5 \%$ & $0 \%$ \\
\hline
\end{tabular}

Fuente: www.spensiones.cl 


\section{METODOLOGÍA Y DATOS}

El presente estudio contempla el análisis del comportamiento de los fondos de pensión para el período comprendido desde la creación de los multifondos, octubre 2002 hasta agosto de 2012. Las administradoras de fondos de pensión estudiadas son Capital, Cuprum, Habitat, Planvital y Provida, ya que son las que han tenido continuidad de operación a lo largo del período de análisis. Los fondos que se analizan son el A, B, C y D de cada AFP; el fondo E no se estudia ya que no posee renta variable en la gran mayoría del período analizado, por lo que el riesgo sistemático tiende a ser nulo.

Para construir la muestra se han considerado las rentabilidades mensuales durante el período de análisis, obtenidas del Boletín Mensual que elabora la Superintendencia de Pensiones (www.spensiones.cl).

Como cartera representativa de mercado se han considerado cinco índices de referencia, de los cuales dos son representativos del mercado de valores chileno y los otros tres del conjunto de fondos de pensiones. El primero considera al Índice de Precios Selectivo de Acciones (IPSA), que contiene las 40 acciones de mayor volumen de transacción. El segundo es el Índice General de Precios de Acciones, IGPA, que aglutina todas las acciones que se transan en la Bolsa de Santiago y se pondera de manera principal por el volumen de transacción ${ }^{1}$. El tercer índice utilizado es el benchmark para el límite máximo (Lím. Máx.) de inversión en renta variable de cada fondo (Tabla 1) y el porcentaje complementario de renta fija. La evolución de la inversión en renta variable se puede observar en el anexo A. El cuarto benchmark está asociado a la composición real mensual de cada fondo agregado en renta variable y renta fija (fondo); en particular, este índice para medición de beta es muy apropiado, ya que contrasta los resultados con un portafolio de la misma composición de riesgo. Por último, y como índice de los fondos riesgosos, se realiza un ponderado (Ponderado) de todos los fondos según la inversión. Cabe mencionar, que dentro de las posibilidades de inversión en renta variable y renta fija a la cual pueden acceder las administradoras de fondos están contempladas las inversiones en el extranjero. En este estudio en particular tanto la renta fija como la variable se caracterizan por indicadores del mercado nacional, lo cual se basa en una comparación restringida a inversiones nacionales, dado que la posibilidad de invertir en el extranjero abre mejores alternativas, por lo que los resultados obtenidos por las administradoras debieran ser mejores.

Más información en www.bolsadesantiago.com. 
Como activo libre de riesgo se consideran los pagarés del Banco Central a 30 días, cuyos valores fueron obtenidos del sitio web del Banco Central de Chile (www. bcentral.cl).

Para la estimación de los coeficientes beta, se utiliza la ecuación 4, mediante mínimos cuadrados generalizados para así tener contemplado la posible existencia de heterocedasticidad y auto-correlación. Este modelo se aplicará para cada fondo de cada AFP analizada, y con cada índice de renta variable definido. Para determinar la consistencia de los parámetros calculados se analiza el comportamiento temporal mediante análisis de estimaciones recursivas (CUSUM y CUSUMQ), ventanas de tiempo solapadas y ventanas de tiempo no solapadas, según la metodología utilizada por García y García (2009).

Para la estimación de los coeficientes beta en períodos no solapados, se divide el período en dos subperíodos de 60 meses, el período más utilizado de cálculo de beta. Para el caso de no solapados, se utilizan periodos de 60 meses con un movimiento trimestral.

Tanto el análisis de errores de las estimaciones recursivas como el de periodos solapados y no solapados se realizan para los índices benchmark IPSA, Fondo y Ponderado.

Para analizar la factibilidad y precisión de la predicción del beta de los fondos de pensiones se comparan tres metodologías: métodos de los mínimos cuadrados, método Blume y método Vasicek. El método de mínimos cuadrado será aplicado para períodos de ajuste de 60 meses para el cálculo del beta. Por otra parte el método Blume (1975) se calculará con base en los 60 meses pasados, separándolos en dos subperiodos de 30 meses cada uno para obtener tendencia del beta. El método Vasicek (1973) también se calculará con base en 60 meses. Los pronósticos de los betas por las tres metodologías se contrastarán con el beta calculado 30 meses después. Para determinar el mejor modelo para pronosticar se utilizarán los criterios de error cuadrático medio, error absoluto medio y error absoluto medio relativo.

\section{ANÁLISIS DE RESULTADOS}

Con las rentabilidades mensuales de los distintos fondos de pensiones evaluados, y con los indicadores de mercado IPSA, IGPA, Máximo Límite, Fondo y Ponderado, se procede a aplicar el modelo CAPM para determinar el riesgo sistemático beta, para el período completo analizado (tabla 2). Se puede observar que los betas para todo el período calculado con base en los índices de mercado IPSA e IGPA son 
menores que uno, lo cual implica una administración defensiva, y tal cual como se puede deducir de las restricciones de inversión, a medida que el fondo es menos riesgoso el beta es menor. Nótese que los betas calculados con IPSA son siempre menores para cada fondo que el calculado con respecto al IGPA; esto demuestra que este último índice es menos riesgoso que el IPSA, lo cual tiene explicación, ya que se compone con todas las acciones, algunas de las cuales tienen muy baja presencia en transacciones.

Al analizar los valores del beta calculados con base en el máximo límite de inversión en renta variable, todos los betas son menores a uno, lo que implica una administración de menor riesgo que lo permitido, medido a través del beta. Los resultados del beta calculado con respecto al límite máximo son bastante importante, ya que independiente del tipo de fondo y de la AFP, todos los betas se encuentran en un rango entre 0,65 y 0,71, lo que muestra una conducta de administración del riesgo consistente con respectos a los límites, que es un $30 \%$ a $35 \%$ menor que el máximo riesgo permitido, medido con el parámetro beta. Al comparar los betas del límite máximo con los obtenidos a través del IPSA y del IGPA, son mayores, lo cual se explica porque el benchmark construido como límite máximo tiene un porcentaje en renta fija, que lo hace ser menos riesgoso que los índices representativos del mercado accionario.

El beta determinado para cada fondo de cada AFP con respecto al índice representativo de cada fondo mensual es interesante para analizar la dispersión entre los diferentes administradores de fondos para cada tipo de fondo. Se puede observar una homogeneidad de los betas y mínimas diferencias entre las administradoras de fondos para cada tipo de fondo. Una de las mayores diferencias se puede observar para la AFP 4 en el fondo tipo D. Por último, mediante el modelamiento con el benchmark de la inversión total de los 4 fondos en el índice ponderado, se puede concluir que contra este índice los fondos tipo A son agresivos, mientras que los demás fondos son defensivos.

Estos resultados son muy similares a los obtenidos en el trabajo de García y García (2009), lo que explica en este caso que la mayor reacción al utilizar índices construidos con parte de renta fija se debe a que se asemejan de mejor forma a la cartera de referencia de los fondos de pensiones, ya que los índices bursátiles (como el IPSA) no consideran la renta fija, que para el caso, es fundamental en gran parte de los fondos en estudio. 
Tabla 2. Riesgo Beta calculado para cada fondo de cada AFP

\begin{tabular}{|c|c|c|c|c|c|}
\hline \multirow{2}{*}{ Fondos } & \multicolumn{5}{|c|}{ Benchmark } \\
\hline & IPSA & IGPA & Lím. Máx & Fondo & Ponderado \\
\hline \multicolumn{6}{|l|}{ Fondo A } \\
\hline AFPl & 0,56 & 0,65 & 0,70 & 0,73 & 1,21 \\
\hline AFP2 & 0,55 & 0,65 & 0,69 & 0,73 & 1,21 \\
\hline AFP3 & 0,56 & 0,66 & 0,70 & 0,73 & 1,22 \\
\hline AFP4 & 0,56 & 0,65 & 0,69 & 0,73 & 1,21 \\
\hline AFP5 & 0,54 & 0,64 & 0,68 & 0,71 & 1,19 \\
\hline \multicolumn{6}{|l|}{ Fondo B } \\
\hline AFPl & 0,41 & 0,48 & 0,68 & 0,73 & 0,89 \\
\hline AFP2 & 0,40 & 0,47 & 0,67 & 0,73 & 0,89 \\
\hline AFP3 & 0,41 & 0,48 & 0,69 & 0,74 & 0,91 \\
\hline AFP4 & 0,40 & 0,47 & 0,67 & 0,73 & 0,89 \\
\hline AFP5 & 0,41 & 0,48 & 0,68 & 0,74 & 0,90 \\
\hline \multicolumn{6}{|l|}{ Fondo C } \\
\hline AFPl & 0,26 & 0,30 & 0,65 & 0,68 & 0,58 \\
\hline AFP2 & 0,26 & 0,30 & 0,66 & 0,69 & 0,59 \\
\hline AFP3 & 0,26 & 0,31 & 0,66 & 0,70 & 0,59 \\
\hline AFP4 & 0,26 & 0,30 & 0,64 & 0,68 & 0,57 \\
\hline AFP5 & 0,26 & 0,31 & 0,66 & 0,70 & 0,59 \\
\hline \multicolumn{6}{|l|}{ Fondo D } \\
\hline AFPl & 0,14 & 0,16 & 0,68 & 0,68 & 0,30 \\
\hline AFP2 & 0,13 & 0,15 & 0,65 & 0,68 & 0,29 \\
\hline AFP3 & 0,13 & 0,15 & 0,64 & 0,67 & 0,29 \\
\hline AFP4 & 0,12 & 0,14 & 0,60 & 0,62 & 0,27 \\
\hline AFP5 & 0,13 & 0,15 & 0,67 & 0,69 & 0,30 \\
\hline
\end{tabular}

Fuente: elaboración propia

Todos los betas calculados para el período completo analizado y mostrados en la tabla 2 son significativos al $1 \%$. Para apreciar la homogeneidad de los betas, se realizó el análisis de la estadística descriptiva de los resultados obtenidos (tabla 3). Se puede observar que para los fondos A, B y C el coeficiente de variación es 
muy bajo, y refleja la homogeneidad en la administración de los fondos, lo que es resultado del efecto manada o rebaño. El fondo D muestra una mayor dispersión, lo que hace pensar una administración menos homogénea, pero al ser menos riesgoso, la rentabilidad es menor, por lo cual las pequeñas diferencias de rentabilidad son un porcentaje de dispersión mayor que en los fondos más riesgosos.

Estos resultados apoyan las conclusiones obtenidas por Raddatz y Schmukler (2011) al analizar las carteras de inversión de las diferentes AFP a través del tiempo.

Tabla 3. Estadística descriptiva de los betas obtenidos

\begin{tabular}{llllll}
\hline \multicolumn{7}{c}{$\begin{array}{l}\text { Benchmark } \\
\text { IPSA }\end{array}$} & IGPA & Lím. Máx & Fondo & Ponderado \\
Media & & & & & \\
Fondo A & 0,55 & 0,65 & 0,69 & 0,73 & 1,21 \\
Fondo B & 0,41 & 0,48 & 0,68 & 0,73 & 0,90 \\
Fondo C & 0,26 & 0,30 & 0,65 & 0,69 & 0,59 \\
Fondo D & 0,13 & 0,15 & 0,65 & 0,67 & 0,29 \\
Desviación Estándar & & & & \\
Fondo A & 0,01 & 0,01 & 0,01 & 0,01 & 0,01 \\
Fondo B & 0,00 & 0,01 & 0,01 & 0,01 & 0,01 \\
Fondo C & 0,00 & 0,00 & 0,01 & 0,01 & 0,01 \\
Fondo D & 0,01 & 0,01 & 0,03 & 0,03 & 0,01 \\
Coeficiente de Variación & & & & \\
Fondo A & $1,02 \%$ & $1,05 \%$ & $1,02 \%$ & $1,01 \%$ & $0,84 \%$ \\
Fondo B & $0,91 \%$ & $1,08 \%$ & $0,91 \%$ & $0,88 \%$ & $0,89 \%$ \\
Fondo C & $1,27 \%$ & $1,27 \%$ & $1,27 \%$ & $1,60 \%$ & $1,52 \%$ \\
Fondo D & $4,86 \%$ & $5,28 \%$ & $4,86 \%$ & $3,82 \%$ & $4,02 \%$ \\
\hline
\end{tabular}

Fuente: elaboración propia

Para analizar la consistencia de los betas calculados se realizó un análisis temporal. Del análisis de las estimaciones recursivas, mediante la herramienta CUSUM, se puede deducir que las estimaciones tienen un alto grado de estabilidad, sin sobrepasar las bandas de confianza del $5 \%$ de nivel de significancia. Los resultados muestran que en la mayoría de los casos, la tendencia de la suma de los residuos recursivos se mantiene con poca oscilación dentro del período de análisis, y al permanecer dentro de las bandas de confianzas de la herramienta, demuestran la estabilidad que arrojan los betas calculados. Sin embargo, aquellas pequeñas 
oscilaciones obligan a buscar otras alternativas para confirmar la estabilidad de los betas. Por este motivo se utilizó la herramienta CUSUMO, la cual muestra de manera más clara los problemas de estabilidad, ya que en ciertos fondos, la gráfica sobrepasa las bandas de significancia del modelo. En particular, para los fondos Cy $\mathrm{D}$, de los distintos planes de pensiones, e independiente del índice de mercado que se utilice, se ve una clara inestabilidad que no se detectó cuando se usó CUSUM. De este modo, y dado que el análisis gráfico no ha sido concluyente, se realiza el análisis de tiempos solapados y no solapados, de acuerdo con lo expuesto en la metodología.

En primera instancia se analizarán los betas para periodos no solapados, lo cual implica dividir el período completo en dos subperíodos de 60 meses cada uno para los índices IPSA, Fondo y Ponderado. Los resultados se pueden observar en la tabla 4; el primer período está comprendido entre octubre 2002 hasta septiembre 2007 y el segundo período entre septiembre 2007 a agosto 2012. Este último período es de interés ya que calza con la caída asociada a la crisis subprime y posteriores problemas económicos de algunos países de la Comunidad Europea. Se puede observar que en todos los fondos, para cada tipo de fondo, el cambio de beta es notorio, y en todos los casos el beta aumentó, lo cual indica el cambio de una política de administración defensiva a una más agresiva. Con estos antecedentes se hace importante el análisis de períodos solapados para observar cómo fue la evolución del beta a través del tiempo.

Tabla 4. Betas para periodos no solapados

\begin{tabular}{|c|c|c|c|c|c|c|}
\hline & \multicolumn{2}{|c|}{ IPSA } & \multicolumn{2}{|c|}{ Fondo } & \multicolumn{2}{|c|}{ Ponderado } \\
\hline & $\begin{array}{l}\text { Oct/02- } \\
\text { Sep/07 }\end{array}$ & $\begin{array}{l}\text { Sep/07- } \\
\text { Ago/12 }\end{array}$ & $\begin{array}{l}\text { Oct/02- } \\
\text { Sep/07 }\end{array}$ & $\begin{array}{l}\text { Sep/07- } \\
\text { Ago/12 }\end{array}$ & $\begin{array}{l}\text { Oct/02 } \\
\text { Sep/07 }\end{array}$ & $\begin{array}{l}\text { Sep/07 } \\
\text { Ago/12 }\end{array}$ \\
\hline \multicolumn{7}{|c|}{ Fondo A } \\
\hline AFPl & 0,30 & 0,68 & 0,40 & 0,89 & 0,68 & 1,42 \\
\hline AFP2 & 0,31 & 0,68 & 0,40 & 0,89 & 0,70 & 1,41 \\
\hline AFP3 & 0,31 & 0,69 & 0,40 & 0,90 & 0,69 & 1,42 \\
\hline AFP4 & 0,30 & 0,68 & 0,39 & 0,89 & 0,67 & 1,41 \\
\hline AFP5 & 0,29 & 0,67 & 0,38 & 0,87 & 0,67 & 1,39 \\
\hline \multicolumn{7}{|c|}{ Fondo B } \\
\hline AFPl & 0,23 & 0,50 & 0,43 & 0,86 & 0,53 & 1,04 \\
\hline AFP2 & 0,23 & 0,49 & 0,44 & 0,85 & 0,55 & 1,02 \\
\hline AFP3 & 0,24 & 0,50 & 0,47 & 0,86 & 0,59 & 1,03 \\
\hline
\end{tabular}




\begin{tabular}{|c|c|c|c|c|c|c|}
\hline & \multicolumn{2}{|c|}{ IPSA } & \multicolumn{2}{|c|}{ Fondo } & \multicolumn{2}{|c|}{ Ponderado } \\
\hline & $\begin{array}{l}\text { Oct/02 } \\
\text { Sep/07 }\end{array}$ & $\begin{array}{l}\text { Sep/07 } \\
\text { Ago/12 }\end{array}$ & $\begin{array}{l}\text { Oct/02 } \\
\text { Sep/07 }\end{array}$ & $\begin{array}{l}\text { Sep/07- } \\
\text { Ago/12 }\end{array}$ & $\begin{array}{l}\text { Oct/02 } \\
\text { Sep/07 }\end{array}$ & $\begin{array}{l}\text { Sep/07- } \\
\text { Ago/12 }\end{array}$ \\
\hline AFP4 & 0,23 & 0,49 & 0,45 & 0,85 & 0,56 & 1,02 \\
\hline AFP5 & 0,23 & 0,50 & 0,44 & 0,86 & 0,55 & 1,04 \\
\hline \multicolumn{7}{|c|}{ Fondo C } \\
\hline AFPl & 0,16 & 0,31 & 0,42 & 0,80 & 0,39 & 0,65 \\
\hline AFP2 & 0,17 & 0,31 & 0,46 & 0,81 & 0,42 & 0,65 \\
\hline AFP3 & 0,17 & 0,31 & 0,47 & 0,81 & 0,43 & 0,65 \\
\hline AFP4 & 0,17 & 0,30 & 0,45 & 0,79 & 0,41 & 0,63 \\
\hline AFP5 & 0,17 & 0,31 & 0,46 & 0,82 & 0,42 & 0,66 \\
\hline \multicolumn{7}{|c|}{ Fondo D } \\
\hline AFPl & 0,12 & 0,14 & 0,52 & 0,78 & 0,26 & 0,30 \\
\hline AFP2 & 0,11 & 0,14 & 0,54 & 0,77 & 0,28 & 0,29 \\
\hline AFP3 & 0,11 & 0,14 & 0,53 & 0,76 & 0,27 & 0,29 \\
\hline AFP4 & 0,10 & 0,13 & 0,49 & 0,71 & 0,25 & 0,27 \\
\hline AFP5 & 0,11 & 0,14 & 0,53 & 0,79 & 0,27 & 0,30 \\
\hline
\end{tabular}

Fuente: elaboración propia

Al analizar la estabilidad de beta a través del tiempo con períodos móviles en cada un trimestre, se puede observar que desde la creación de los multifondos los betas han aumentado, al analizarlos con respecto al IPSA (gráfico 1). Esto es un hecho que se puede apreciar para todos los tipos de fondos. También se puede notar que en marzo del 2009 todos los fondos comienzan a tener una estabilidad en general, con una pequeña tendencia a la baja, la cual se ve más marcada en el fondo D. Este mismo hecho se puede apreciar para el cálculo del beta con respecto al índice ponderado (anexo B).

Por último, se analizó en períodos no solapados el beta calculado con respecto al índice de cada fondo, para cada AFP, y también cómo evoluciona el coeficiente de variación a través del tiempo. Del análisis de los fondos tipo A (gráfico 2), se puede apreciar que el efecto manada es una constante a través del tiempo, los betas de los 5 fondos siempre se mueven en conjunto. El máximo valor que alcanza el coeficiente de variación es 1,80 \%, al comienzo y en marzo de 2009. Posterior a junio de 2009 se ve una estabilización de los betas y del coeficiente de variación para los fondos tipo A. Los demás tipos de fondos se reportan los gráficos en los anexos C, D y E. Para el caso de los fondos tipo B se aprecia el efecto manada, y un descenso del 
coeficiente de variación y la estabilidad con un valor menor al 1 \% desde diciembre del 2008 hasta la actualidad. En el caso de los fondos tipo C sucede lo mismo con el coeficiente de variación, solo que se estabiliza bajo $3 \%$. En los fondos del tipo E se nota una mayor dispersión, y una tendencia a la estabilización del coeficiente de variación, también posterior a diciembre 2008. Dado que para todos los fondos existe una estabilización del coeficiente de variación asociado al beta en diciembre del 2008, esto implica que desde enero del 2004 comenzó a existir una mayor homogeneización de los fondos de cada tipo, ya que para el cálculo se utilizan 60 meses. Por otro lado, esto también permite concluir que existió un periodo de 4 trimestres de ajuste, posterior a la creación de los multifondos en octubre del 2002.

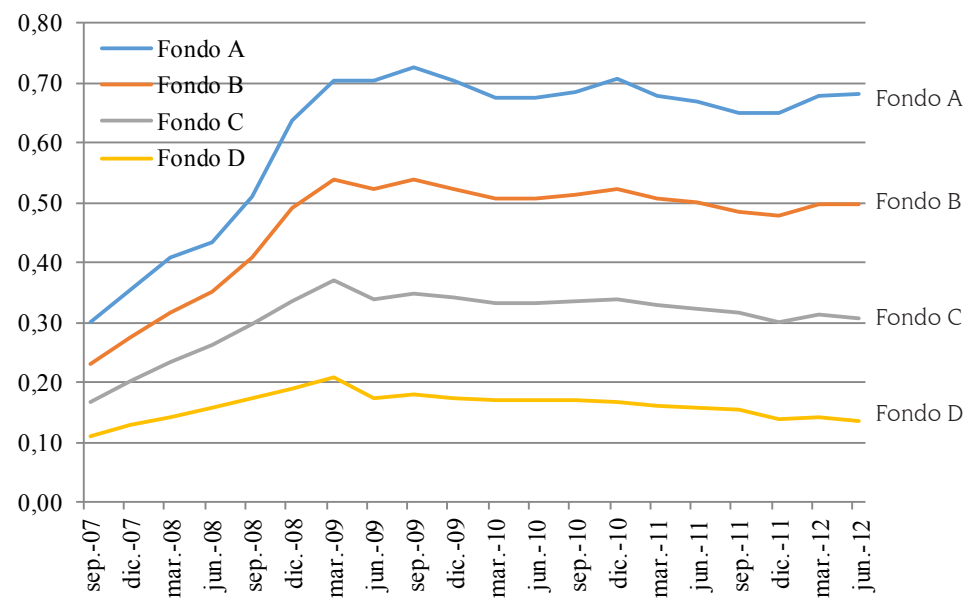

Gráfico 1. Evolución de beta de los tipos de fondos con respecto al IPSA Fuente: elaboración propia

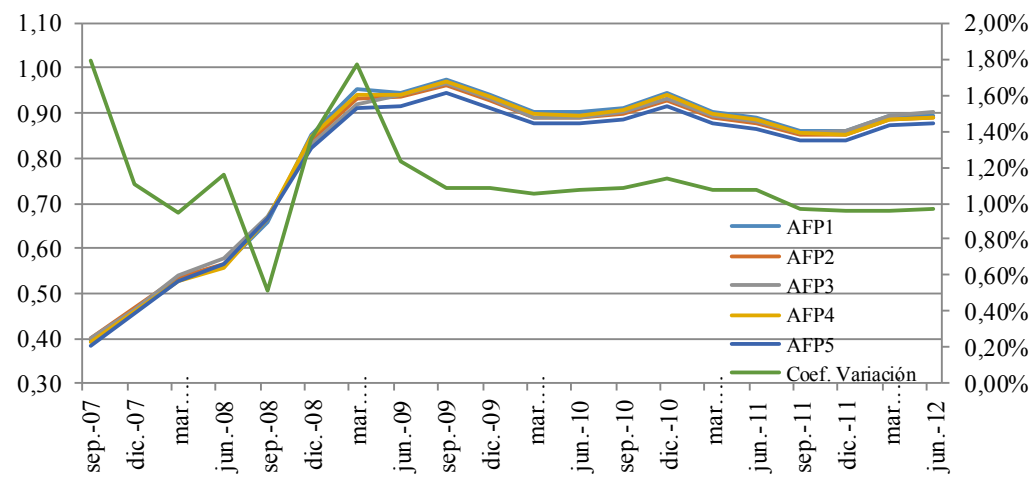

Gráfico 2. Evolución de beta de los fondos tipo A con respecto al fondo.

Fuente: elaboración propia 
Por último, cabe destacar que en la mayoría de las metodologías expuestas, el coeficiente beta muestra una estabilidad que hace posible la utilización como predictor para una tendencia de riesgo, esto es, para definir si un fondo es agresivo o defensivo, posterior a diciembre del 2008. En general, se aprecia una estabilidad del riesgo beta, un tanto diferente a los resultados obtenidos por García y García (2009), en donde se tiene una cierta irregularidad en la tendencia para los planes de pensiones del sistema individual de España.

El cálculo de los pronósticos a través de las tres metodologías utilizadas por García y García (2009) se realizará desde enero 2004 donde comienza una estabilización de los fondos después de la puesta en marcha de los multifondos en octubre del 2002. Las estimaciones se harán con el benchmark de mercado IPSA.

La conclusión que se puede obtener, posterior al haber realizado este análisis profundo del beta de los fondos de pensiones chilenos para el período octubre 2002 agosto 2012, es que existe una política de administración defensiva de mínimos cuadrados, lo cual queda reflejado en los tres indicadores de bondad de predicción, ya que obtiene los menores valores para todos los tipos de fondos y todos los fondos de cada tipo (tabla 5). El método de Blume, que toma la tendencia de cambio de los betas en subperiodos consecutivos, no tiene buenos pronósticos porque el periodo de predicción tiene una parte de ajuste o puesta en marcha de los multifondos, ya que si bien se comenzó a analizar en enero del 2004, no quedó de manera completa fuera los últimos meses de ajuste que afectan al primer subperíodo utilizado por este modelo.

En general, los mejores pronósticos se realizan para los fondos del tipo B seguidos por los del tipo C; esto quiere decir que estos fondos son los más predecibles a través de los modelos analizados, en particular por los mínimos cuadrados y el método Vasicek. Por el contrario, los fondos tipo D, los de menor riesgo analizados, son los que a través del método de Vasicek tienen mayor error al momento de pronosticar el beta.

\section{CONCLUSIONES}

Una conclusión que se puede obtener, posterior al haber realizado este análisis profundo del beta de los fondos de pensiones chilenos para el período octubre 2002 agosto 2012, es que existe una política de administración defensiva, ya que los betas obtenidos para todos los fondos con respecto a los índices del mercado bursátil (IPSA e IGPA) son menores que 1. Sin duda que esta conclusión reafirma la forma de administración de portafolios, y su objetivo de la administración y del uso del dinero para pensiones, o sea, una visión de mediano y largo plazo. 
Tabla 5. Análisis de Error generado por pronósticos de betas para cada fondo por tipo de fondo

\begin{tabular}{|c|c|c|c|c|c|c|c|c|c|}
\hline & \multicolumn{3}{|c|}{ Error Cuadrado Medio } & \multicolumn{3}{|c|}{ Error Absoluto Medio } & \multicolumn{3}{|c|}{ Error Absoluto Relativo Medio } \\
\hline & $\mathrm{MCO}$ & Blume & Vasicek & $\mathrm{MCO}$ & Blume & Vasicek & $\mathrm{MCO}$ & Blume & Vasicek \\
\hline \multicolumn{10}{|c|}{ Fondo A } \\
\hline AFPl & 0,04 & 2,07 & 0,07 & 0,00 & 4,30 & 0,01 & $5,01 \%$ & $301,89 \%$ & $9,55 \%$ \\
\hline AFP2 & 0,04 & 2,01 & 0,07 & 0,00 & 4,05 & 0,01 & $5,20 \%$ & $295,98 \%$ & $9,90 \%$ \\
\hline AFP3 & 0,04 & 1,96 & 0,08 & 0,00 & 3,84 & 0,01 & $6,45 \%$ & $287,17 \%$ & $11,15 \%$ \\
\hline AFP4 & 0,04 & 2,03 & 0,07 & 0,00 & 4,11 & 0,01 & $5,21 \%$ & $296,83 \%$ & $9,75 \%$ \\
\hline AFP5 & 0,04 & 1,96 & 0,07 & 0,00 & 3,85 & 0,01 & $5,47 \%$ & $293,11 \%$ & $10,12 \%$ \\
\hline \multicolumn{10}{|c|}{ Fondo B } \\
\hline AFPl & 0,02 & 1,47 & 0,03 & 0,00 & 2,16 & 0,00 & $3,62 \%$ & $289,67 \%$ & $5,87 \%$ \\
\hline AFP2 & 0,02 & 1,48 & 0,03 & 0,00 & 2,19 & 0,00 & $3,20 \%$ & $292,93 \%$ & $5,36 \%$ \\
\hline AFP3 & 0,02 & 1,47 & 0,03 & 0,00 & 2,16 & 0,00 & $3,27 \%$ & $287,63 \%$ & $5,96 \%$ \\
\hline AFP4 & 0,02 & 1,47 & 0,03 & 0,00 & 2,15 & 0,00 & $3,16 \%$ & $290,78 \%$ & $5,09 \%$ \\
\hline AFP5 & 0,02 & 1,46 & 0,03 & 0,00 & 2,13 & 0,00 & $3,31 \%$ & $288,46 \%$ & $5,83 \%$ \\
\hline \multicolumn{10}{|c|}{ Fondo C } \\
\hline AFPl & 0,01 & 0,89 & 0,03 & 0,00 & 0,79 & 0,00 & $3,40 \%$ & $275,35 \%$ & $8,93 \%$ \\
\hline AFP2 & 0,01 & 0,96 & 0,03 & 0,00 & 0,91 & 0,00 & $4,35 \%$ & $285,97 \%$ & $9,20 \%$ \\
\hline AFP3 & 0,02 & 0,94 & 0,03 & 0,00 & 0,88 & 0,00 & $4,71 \%$ & $282,70 \%$ & $10,38 \%$ \\
\hline AFP4 & 0,02 & 0,92 & 0,04 & 0,00 & 0,84 & 0,00 & $5,51 \%$ & $284,13 \%$ & $11,87 \%$ \\
\hline AFP5 & 0,01 & 0,94 & 0,03 & 0,00 & 0,89 & 0,00 & $4,48 \%$ & $286,02 \%$ & $10,13 \%$ \\
\hline \multicolumn{10}{|c|}{ Fondo D } \\
\hline AFPl & 0,02 & 0,39 & 0,12 & 0,00 & 0,15 & 0,01 & $14,84 \%$ & $239,26 \%$ & $73,77 \%$ \\
\hline AFP2 & 0,03 & 0,45 & 0,13 & 0,00 & 0,21 & 0,02 & $19,06 \%$ & $275,15 \%$ & $79,86 \%$ \\
\hline AFP3 & 0,03 & 0,42 & 0,13 & 0,00 & 0,18 & 0,02 & $17,95 \%$ & $260,77 \%$ & $81,39 \%$ \\
\hline AFP4 & 0,03 & 0,38 & 0,14 & 0,00 & 0,15 & 0,02 & $19,74 \%$ & $252,92 \%$ & $93,39 \%$ \\
\hline AFP5 & 0,03 & 0,43 & 0,13 & 0,00 & 0,18 & 0,02 & $17,50 \%$ & $259,03 \%$ & $79,35 \%$ \\
\hline
\end{tabular}

Fuente: elaboración propia

Al analizar los valores del beta calculados con base en el máximo límite de inversión en renta variable, todos se sitúan entre 0,65 y 0,71, lo cual indica una administración de menor riesgo que lo permitido, medido a través del beta. 
En general se evidenció una homogeneidad de los betas de fondos de un mismo tipo, lo que caracteriza el efecto manada o rebaño de la administración de estos fondos.

Al dividir el período analizado en dos subperíodos (el primer período está comprendido entre octubre 2002 hasta septiembre 2007 y el segundo período entre septiembre 2007 a agosto 2012), se pudo observar el cambio de administración de los fondos: desde una muy defensiva a una menos defensivas, todos los fondos aumentaron su beta. Este hecho se puede explicar por el período inical de puesta en marcha de los multifondos y su administración y también la dferencia con el segundo período que calza con con la caída de los valores asociada a la crisis subprime y posteriores problemas económicos de algunos países de la Comunidad Europea.

Del cálculo de los pronósticos, a través de tres metodologías utilizadas, se obtuvo que el método que genera la mejor predicción es el de mínimos cuadrados, para todos los fondos de cada tipo, y medido por tres indicadores de bondad de predicción. Los mejores pronósticos se realizan para los fondos del tipo B seguidos por los del tipo C.

\section{BIBLIOGRAFÍA}

Arrau, P. y Chumacero, P. (1998). Tamaño de los fondos de pensiones en Chile y su desempeño financiero. Cuadernos de Economía, 35(105), pp. 205-235.

Blume, M. E. (1975). Betas and their regression tendencies. Journal of Finance, 30(3), pp.785-795.

Chile (1980). Decreto de Ley 3.500, Establece Nuevo Sistema de Pensiones. 15 títulos.

Fama, E. y French, K. (1992). The Cross-Section of Expected Stock Returns. Journal of Finance, 47(2), pp. 427-465.

Fernández, V. (2005). El Modelo CAPM para Distintos Horizontes de Tiempo. Revista Ingeniería de Sistemas, 19, pp. 7-18.

García, Y. y García, J. (2009). El riesgo beta de los planes de pensiones del sistema individual en España. Innovar, 19(33), pp. 91-104.

Kothari, S. y Shanken, J. (1995). In defense of beta. Journal of Applied Corporate Finance, 8, pp. 53-58.

Litner, J. (1965). The valuation of risk asset and the selection of risky investment in stock portfolios and capital budgets. Review of Economics and Statistics, 47, pp. 13-37.

Markovitz, H. (1952). Portfolio Selection. Journal of Finance, 7(1), pp. 77-91.

Mittelstaedt, F. y Olsen, J. (2003). An Empirical Analysis of the Investment Performance of the Chilean Pension System. Journal of Pension Economics and Finance, 2(1), pp. 7-24. 
Mossin, J. (1966). Equilibrium in a Capital Asset Market. Econometrica, 34(4), pp. 768-783.

Olivares, J. (2005). Investment Behavior of the Chilean Pension Funds. Financial Management Association European Conference, Paper 360419, 56 pp.

Opazo, L., Raddatz, C., y Schmukler, S. (2009). The Long and the Short of Emerging Market Debt. World Bank Policy Research, Working Paper 5056, 56 pp.

Raddatz C. y Schmukler S. (2011) Deconstructing herding: evidence from pension fund Investment behavior. World Bank Policy Research, Working Paper 5700, 54 pp.

Santillán, R., López, D. y Montenegro, J. (2010). Las Administradoras de Fondos de Pensiones y el desarrollo del mercado de capitales en Chile. Ensayos Revista de Economía-Volumen XXIX, 2, pp. 53-76.

Sharpe, W. (1964) Capital Asset Prices: A Theory of Market Equilibrium Under Conditions of Risk. Journal of Finance, 19(3), pp. 425-442.

Vasicek, O. (1973). A note on using cross-sectional information in bayesian estimation of security betas. Journal of Finance, 28(5), pp. 1233-1239.

Zurita, S. y Jara, C. (1999). Desempeño Financiero de Fondos de Pensiones. Estudios Públicos, 74, pp. 227-254. 


\section{ANEXOS}

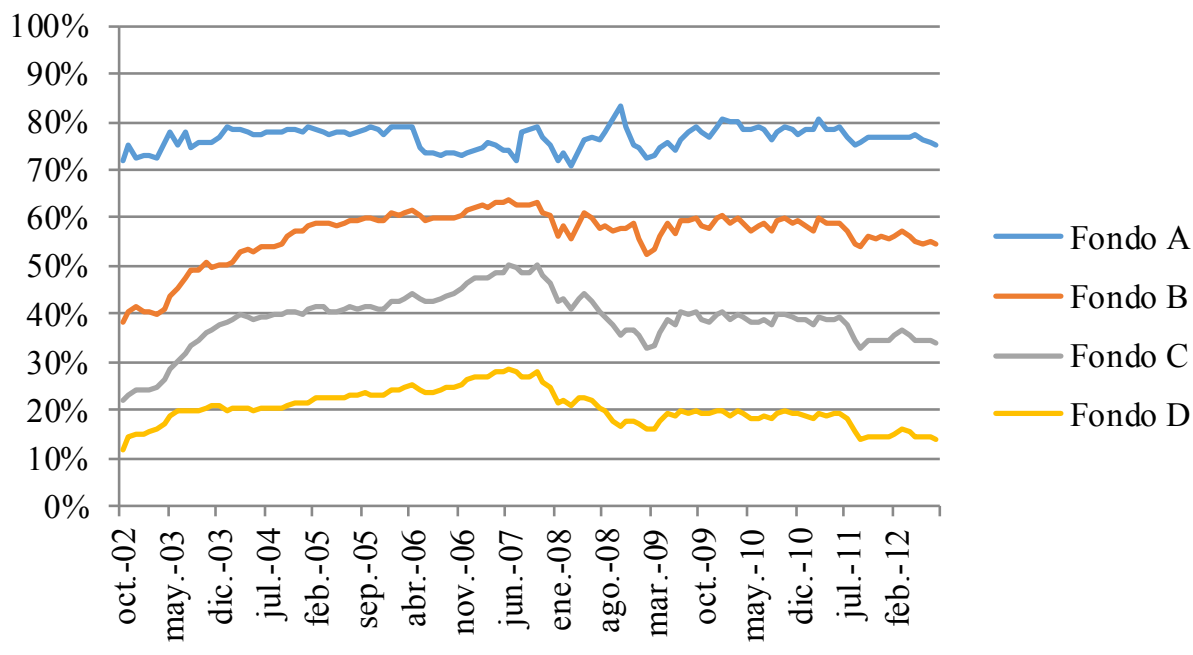

Anexo A. Evolución del porcentaje de inversión en Renta Variable por Fondo.

Fuente: elaboración propia

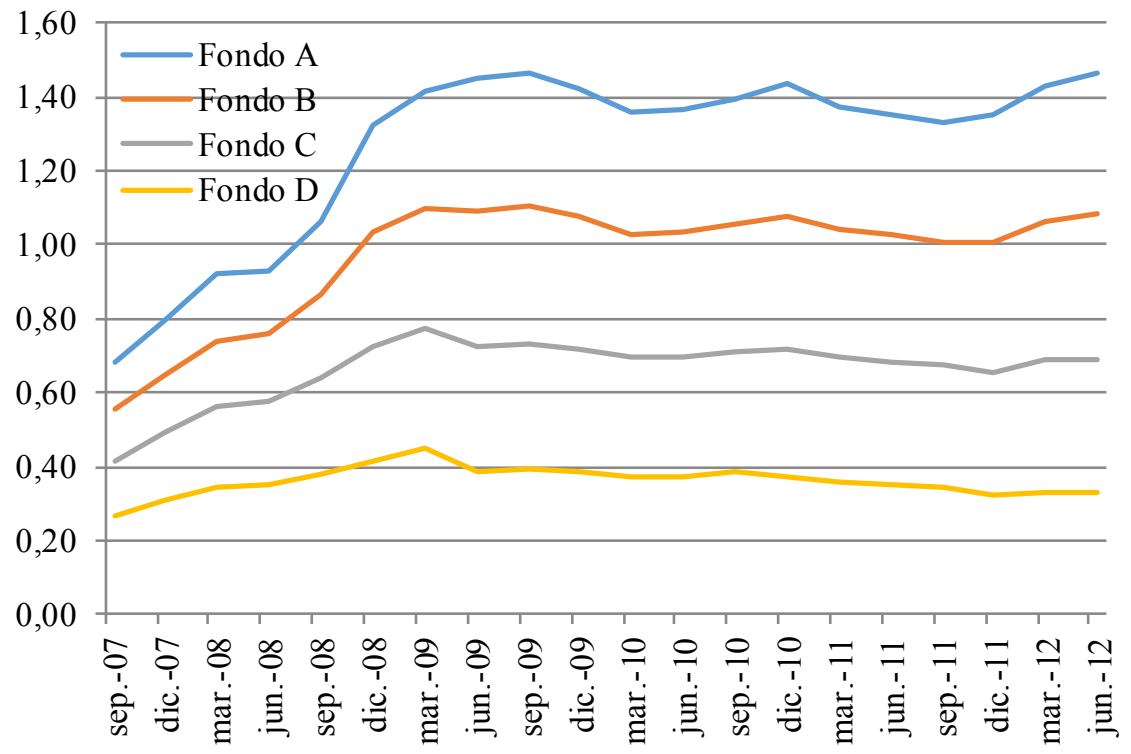

Anexo B. Evolución de beta de los tipos de fondos con respecto al índice ponderado Fuente: elaboración propia 


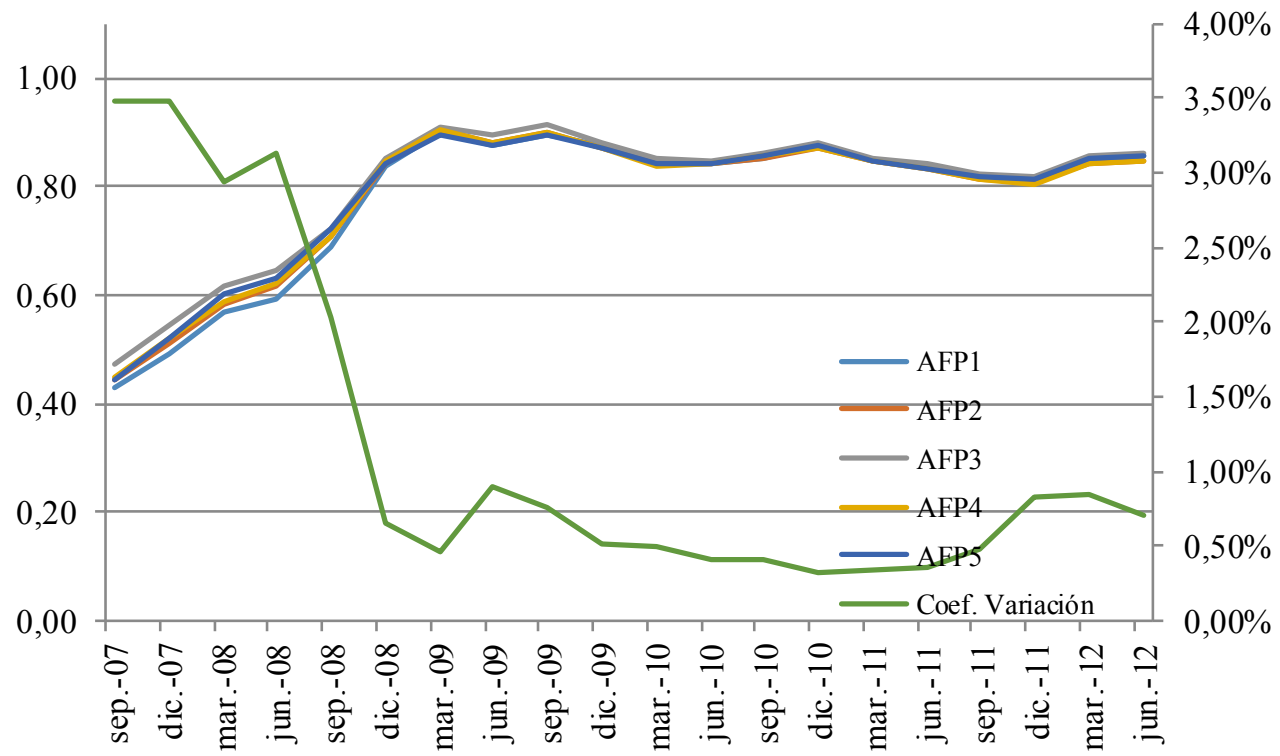

Anexo C. Evolución de beta de los fondos tipo B con respecto al fondo Fuente: elaboración propia

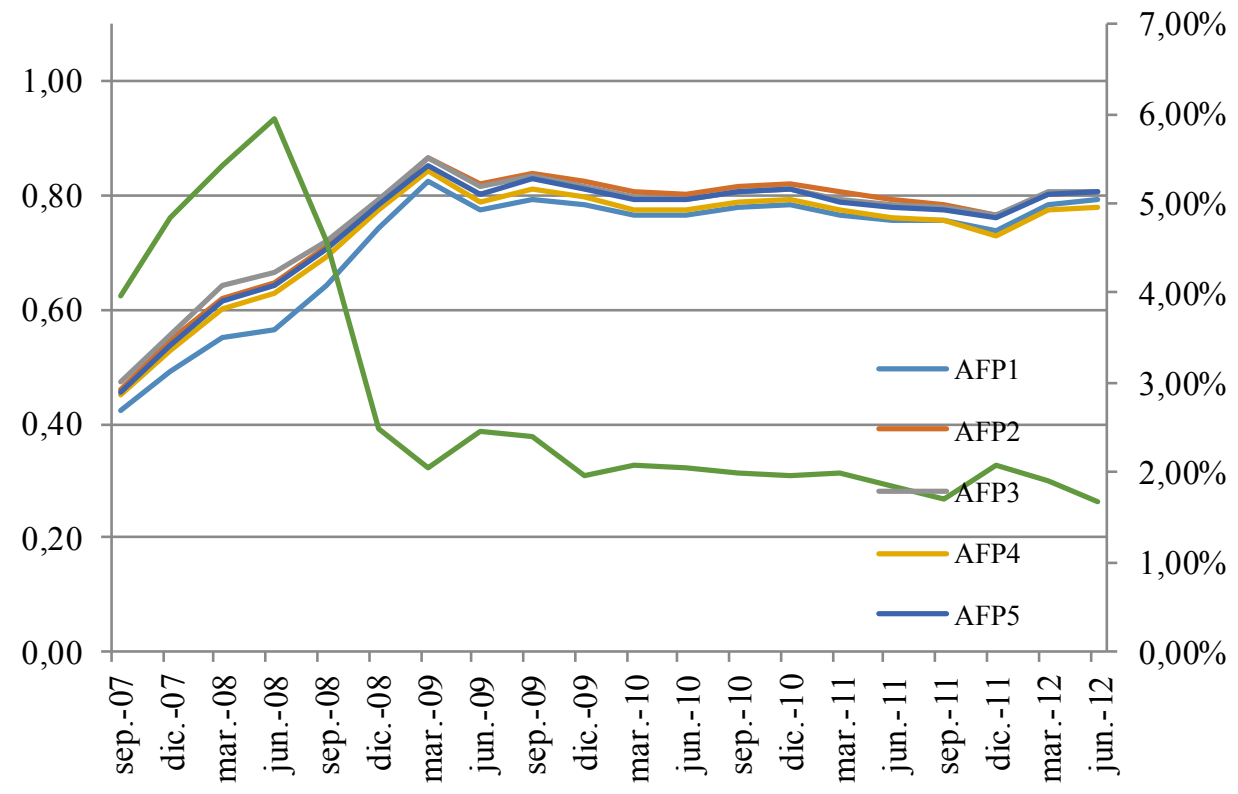

Anexo 4. Evolución de Beta de los Fondos tipo C con respecto al fondo Fuente: elaboración propia 


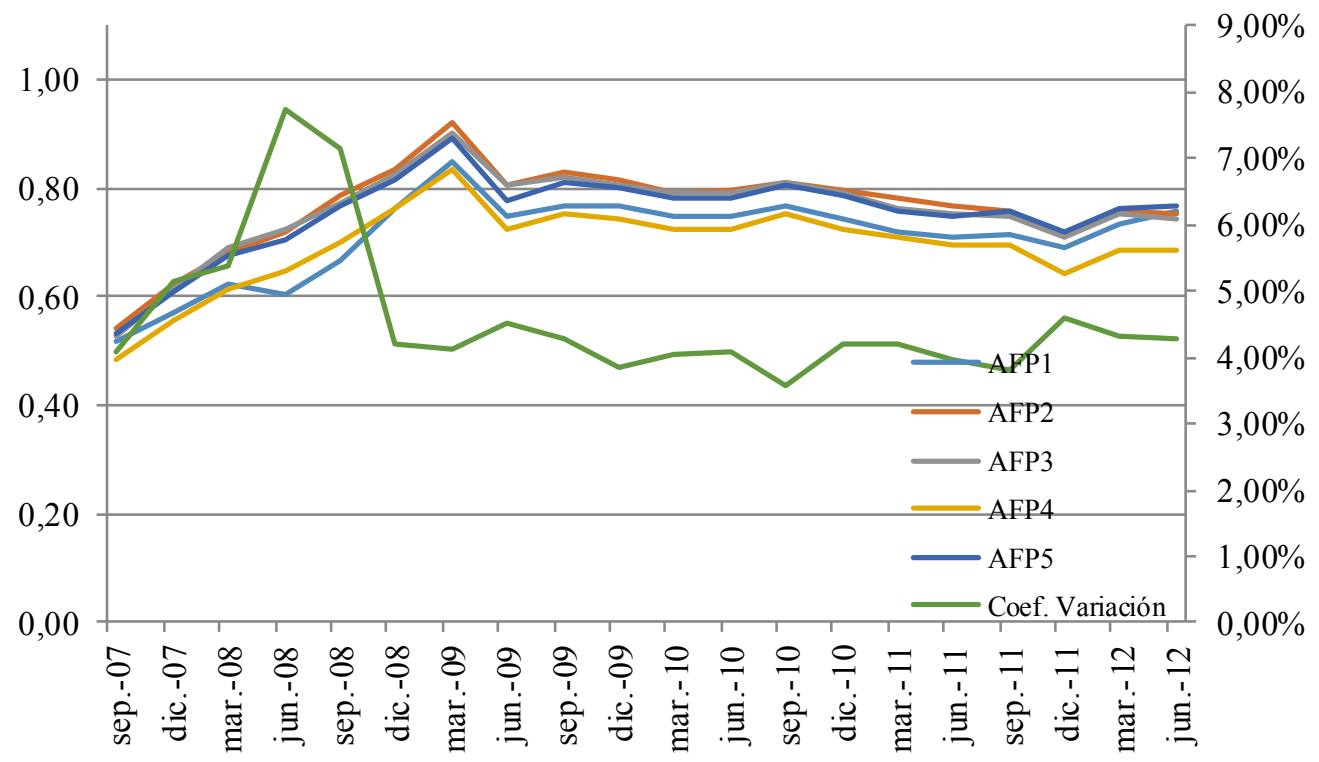

Anexo 5. Evolución de beta de los fondos tipo D con respecto al fondo

Fuente: elaboración propia 